\title{
Geschichte der Architektur der Geschichte
} Überlegungen zur Historisierung einer Disziplin

Christian Freigang

Anlässlich der ersten Olympischen Spiele der Neuzeit begibt sich der französische Schriftsteller und Journalist Charles Maurras 1896 von Paris nach Athen. Der daraus entstandene Text Anthinea. D'Athènes à Florence ist jedoch weit davon entfernt, die sportlichen Ereignisse zu kommentieren. Vielmehr gerät er zu einem ganzheitlichen Kulturentwurf, der in Form eines Reiseberichts die unvergänglichen Qualitäten des griechischen Geistes, des esprit grec, vermittelt und feiert. ${ }^{1}$ Dieser äußere sich in allen Erscheinungsformen der mittelmeerischen Welt zwischen Griechenland, Italien und der Provence in den Geländeformationen, den Sitten der einst wie heute dort lebenden Menschen und vor allem in den Überresten der griechischen Antike. Der geographische Parcours von Osten nach Westen sei zugleich ein zeitlicher - er reicht von der idealen Antike bis in die dekadente, nach den Prinzipien des griechischen Geistes zu reformierende Gegenwart. Die attische Welt, in der alle Erscheinungen sich zu einem harmonischen Ganzen ergänzten, müsse mit allen Organen wahrgenommen werden können, rational erkennend und sinnlich fühlend zugleich. In einer Schlüsselstelle des Reiseberichts macht der Erzähler sich nach langer Vorbereitung daran, die Akropolis als das Spitzenwerk des griechischen Geistes zu betreten. Vor den ersten Säulen der Propyläen, dem Eingangstor der Burg, hält er inne:

1 | Vgl. Maurras, Charles: Anthinea. D’Athènes à Florence, Paris: Juven 1901, wieder abgedruckt in: Ders., Oeuvres capitales. Bd. 1: Sous le signe de Minerve, Paris: Flammarion 1954, S. 167-257. 
Auch wenn ich zugebe, dass ich dabei keine Träne vergossen habe, kann ich es wagen niederzuschreiben, was dann folgte? Über dieser Säule, der ersten, der ich im Chor der jungen Propyläerinnen gewahr geworden war, breitete ich die Arme weit im Raum aus, so weit ich konnte, neigte den Kopf - nicht ohne Vorsicht, musste ich doch wegen einer Truppe von rasch sich nähernden lärmenden Amerikanern vorgeben, den Säulenumfang zu vermessen - und küsste sie wie eine Geliebte mit meinen Lippen. ${ }^{2}$

In dem Säulenstumpf entäußert sich für Maurras gleichsam das gesamte Menschentum. Dieses architektonische Element, Resultat von gesetzmäßiger Ordnung, Proportion, Einheit und Vernunft, schön, sinnlich und verführerisch zugleich, deutet er als Resultat von belebter Vernunft: erregend und immer jung, wie die niemals alternden griechischen Götter in Stein, unsterblich in alle Ewigkeit.

Auch wenn es sich bei dieser Passage nicht um einen Ausschnitt aus einem architekturtheoretischen oder fachgeschichtlichen Werk handelt, sondern um ein literarisch geformtes Motiv, um eine von vielen Variationen prinzipiell vergleichbarer Darstellungen über das Erlebnis der Athener Akropolis, tritt hier ein Stück Architekturinterpretation um 1900 im literarischen und intellektuellen Diskurs der Zeit auf, das auf wichtige Zusammenhänge zwischen literarischer Metaphorik, der zeitgenössischen Architekturdebatte und der Baupraxis verweist. Die Passage enthält in konzentriertester Form vielschichtige Vorstellungen, die mit Reformbestrebungen um 1900 verbunden sind. Insbesondere ist hier eine Schönheitsauffassung zu finden, die Schönheit in ihrem objektiven Sein wie ihrem subjektiven Erleben, rational und emotional als perfekte Einheit zwischen Form und Inhalt begreift. Vernunftgemäße proportionale Ordnung und sinnlich belebte Reaktion beschreiben zwei essenzielle Aspekte eines idealen Weltgesetzes, das Maurras als ganzheitliches Prinzip für sämtliche Bereiche des Lebens versteht. Der klassische Geist repräsentiere eine harmonisch geordnete, mit freudiger Emphase gelebte gesellschaftliche Ordnung. Ein idealer, und das heißt im Sinne Maurras', ein autoritärer und xenophober Staat müsse darauf ausgerichtet sein, dieses Weltgesetz des klassischen Geistes auf immer zu verinnerlichen und zu verteidigen. Die Architektur - selbst in ihrem ruinösen Zustand auf der Akropolis - manifestiere in perfekter und dauerhafter Weise dieses

2 | Ebd., S. 192 [Übersetzung Ch. F.]. 
Weltprinzip, das durch Raum und Zeit Geltung beanspruche. Denn die antiken Säulen seien eben nur dann ewig jung, gleichsam unsterblich, lächeln jugendlich und voller Grazie, wenn und solange die Erbin dieses Geistes - keine andere als die französische Nation - sich auf immer diesen Prinzipien verschreibe.

In Maurras' Erlebnis der sinnlichen antiken Säule schimmert die Haltung einer bedeutenden politischen und gesellschaftlichen Bewegung durch, die eng mit bestimmten Kontexten der Zeit verbunden ist, angefangen von der klassizistischen Gegenbewegung zum Jugendstil bis hin zur Entstehung einer rechtsextremen, scharf antisemitischen Gegenmoderne, die - aufbauend auf derartigen politischen Ästhetizismen Grundlage beziehungsweise essenzieller Bestandteil der europäischen Faschismen werden wird.

Die hier geschilderte Episode mag andeuten, wie Architekturgeschichte (auch) geschrieben werden kann: Nicht allein im sich selbst genügenden kennerschaftlichen Einordnen alter Gebäude, in motivgeschichtlichen »Ableitungen«, technikgeschichtlichen Bezugnahmen oder in biographisch ausgerichteten Erzählungen, sondern im Blick auf das Überblenden, Gegeneinanderstellen und sich Durchdrungensein von historischer Architekturproduktion und -theorie, Metaphern, Denkbildern und Gefühlswelten, kulturellen Praktiken und Zeichensystemen. Die klassizistische Moderne des 20. Jahrhunderts ist nicht eine unschuldige Spielart eines klassischen Paradigmas, das ein harmonisches Ineinsfallen von Form und Inhalt immer wieder neu erprobt. ${ }^{3} \mathrm{Ganz}$ im Gegenteil ist diese klassizistische Moderne - als deren Inkunabel das Théâtre des Champs Elysées in Paris (1911-1913) gelten kann (Abb. 1) - eine Position, die vorgibt, die Auflösung des Mythos in der Moderne zu überwinden, also die Dissoziierung von Individuum, Gesellschaft, Nation, Religion, Kunst und Technik durch ganzheitliche und vitalistische Lebensentwürfe rückgängig zu machen, indem sie bestimmte Diskurshierarchien, Zeichensysteme und Wahrnehmungsweisen als ewig oder überzeitlich gültig fortschreibt. ${ }^{4}$

3 | Vgl. Borsi, Franco: Die monumentale Ordnung. Architektur in Europa 19291939, Stuttgart: Hatje 1987.

4 | Vgl. Freigang, Christian: Auguste Perret, die Architekturdebatte und die "Konservative Revolution" in Frankreich 1900-1930, Berlin/München: Deutscher Kunstverlag 2003. 
Abb. 1, Auguste Perret: Théâtre des Champs Elysées, Fassade, Paris 1911-1913.

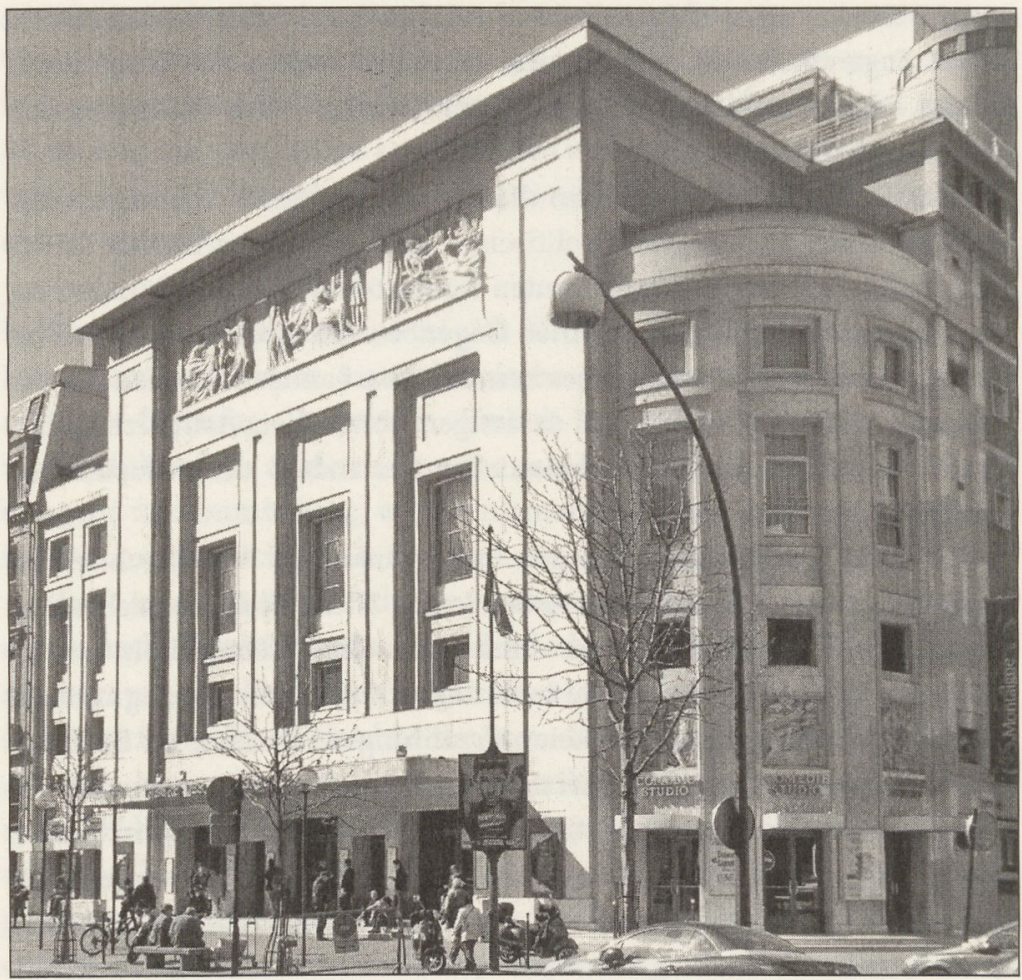

Photographie: Christian Freigang.

Es handelt sich im Speziellen darum, die repräsentationale Leistung dieser Architektur - den programmatischen Bezug auf die griechische Antike - nicht nur als bloße formale Referenz auf bestimmte Bauten beziehungsweise deren Prinzipien zu beschreiben, sondern die chronologische Relation zu verdeutlichen, in der das Heute und das Einst aufeinander bezogen sind. In der Propyläen-Anekdote verdichtet sich nämlich eine spezifische Geschichtsauffassung: Maurras' Evokation der sinnlichen Jugend der antiken Säulen ist eingebettet in eine harsche Kritik an szientistisch-archäologischen Erforschungen der antiken Architektur und Kunst, also an den historischen Wissenschaften mit ihren kleinteiligen Grabungskampagnen und mühevollen, akribischen Textentzifferungen. Derartigen Ansätzen wird zur Last gelegt, ihr Thema zu wenig intuitiv, emotional und seelisch zu erfassen. Ebenso ist Maurras' 
Position als eine Kritik an scheinbar beliebigen, individuellen Vorlieben folgenden Stilzitaten der Zeit des architektonischen Historismus im 19. Jahrhundert zu lesen.

Was wir anhand von Maurras im Speziellen, aber ebenso innerhalb der zeitgenössischen Architekturtheorie und -produktion im Allgemeinen sehen können, sind grundsätzlich unterschiedliche Vorstellungen davon, in welcher Art architektonische Repräsentation - etwa durch die formale Referenz auf Vorbilder und Bauprinzipien - chronologische und historische Logiken vergegenwärtigt. Welche Zeit- und Geschichtsauffassungen werden durch Gebäude und ihre diskursiven Verortungen offengelegt beziehungsweise durch sie konstruiert - als »Architektur der Geschichte«? Maurras' Theorie etwa hebt Geschichte in einer Überzeitlichkeit auf, die einzig ewige Aktualität - Jugend - akzeptiert. Davon kann eine archetypische Auffassung der Geschichte unterschieden werden, die nur die großen, überindividuellen Strömungen und Themen kennt und mit Intuition und Emphase verinnerlicht werden soll. Dies wiederum setzt sich etwa von einem positivistisch-empirischen Zugang und einer scheinbar interesselosen Geschichtsschreibung oder aber von einer Auffassung ab, die auf didaktisch-moralische Umsetzung der Lehren aus der Geschichte Wert legt und sie an Exempeln entwickelt. Solche unterschiedlichen Auffassungen werden in der Geschichtswissenschaft seit dem 19. Jahrhundert in verschiedenen Ausprägungen implizit oder explizit diskutiert. Bemerkenswert ist nun aber - und deswegen eignet sich die Maurras-Passage besonders gut als Beispiel -, dass die Diskurse zu Geschichtsauffassungen keineswegs bloß gelehrte Debatten darstellen, sondern weit in alle Lebensbereiche eindringen, eben auch in die Architektur. - Und es wird nachgerade als deren Aufgabe verstanden, die lebensweltliche Umgebung zu schaffen, in der die jeweilige Geschichtsauffassung Wirklichkeit wird. Architekturgeschichte schreiben heißt somit auch, die damit korrelierten Geschichtsauffassungen mitzudenken. Das gilt schon deshalb, weil Geschichte, geschichtliches Denken und Fühlen ihrerseits keineswegs für alle Zeiten und Räume gültige Konstanten sind. Und das hat jeweils Konsequenzen für die Architektur wie für ihre geschichtliche Verortung, wie in der folgenden, kursorischen »Geschichte der Architektur der Geschichte« zu zeigen sein wird. 


\section{Die Idee EINER UNiversalgeschichte}

Generell bildet sich das Konzept der »Geschichte«als Kollektivsingular zahllose Einzelgeschichten bündeln sich zu einem Ereigniszusammenhang - erst im 18. Jahrhundert aus. Erst seit dieser Zeit wird Geschichte als Begebenheit betrachtet, die von einem Beobachter zu ergründen und $\mathrm{zu}$ verstehen ist, um daraus Anweisungen für politisches, soziales und moralisches Verhalten abzuleiten. Geschichte, vom Menschen bewirkt und auf den Menschen bezogen, wird nun als unendlich verstanden, als Kontinuum eines mehr oder weniger kausal zusammenhängenden Geflechts von Begebenheiten, das unabhängig von göttlichem oder mythischem Wirken funktioniert. Die Geschichte wird Subjekt ihrer selbst, wenn etwa Hegel ein Arbeiten der Weltgeschichte annimmt, oder, allgemeiner verbreitet, bestimmte Ereignisse als Ergebnis »der Geschichte« geltend gemacht werden.

Innerhalb der Geschichte als Gesamtzusammenhang lassen sich nunmehr Historien erzählen, etwa die Geschichte des Handels, der Religion, der Arbeiterbewegung, der Kunst oder eben der Architektur. Der Motor allerdings, der die Geschichte antreibt, kann ganz unterschiedlich bestimmt werden. Das Bewusstsein davon, wie essenziell solche großen, zumeist fundamental konkurrierenden Erzählungen sind, bildet sich im 19. Jahrhundert heraus, also in der Zeit, in der eine säkulare bürgerliche Gesellschaft und ihre Fraktionierungen Legitimations- und Deutungsmuster suchen. Bemühungen beispielsweise um die Geschichte des dritten Standes, die psychologische Geschichte des Lebens Jesu Christi, die Geschichte einer Nation oder die Geschichte der mittelalterlichen Architektur künden davon. All das braucht Gelehrsamkeit, um die widerständigen und fragmentierten Quellen systematisch zu ordnen, zu rekonstruieren und zu interpretieren.

Die Leitdisziplin der sich professionalisierenden und ausweitenden Universität der ersten Hälfte des 19. Jahrhunderts ist die Geschichtswissenschaft. Dazu zählen auch die Kunst- und Architekturgeschichte und in der Folge ein neuer Umgang mit historischen Denkmälern, sowohl in der Denkmalpflege als auch in der Architekturausbildung und -produktion: »Nur Geschichte allein ist zeitgemäß «, ${ }^{5}$ lautet das Motto -

5 | Das Motto, ursprünglich Teil eines Bildkommentars des Malers Wilhelm Kaulbach, ist dem Titel des folgenden Tagungsbandes entnommen: Brix, Michael/ 
schließlich gibt es im 19. Jahrhundert kaum ein Bauwerk, das nicht historistisch konzipiert ist. Dieser Historismus gerät bereits im Laufe des 19. Jahrhunderts in eine Krise, die in dem Umstand begründet liegt, dass die Suche nach den Verläufen der Geschichte weder naturwissenschaftliche Regeln noch moralische oder politische Handlungsanweisungen hervorbringen kann. Insofern scheint Geschichte keine Relevanz für die Gegenwart zu haben. Der gelehrte Historiker wird nunmehr im übertragenen wie im buchstäblichen Sinne zum verstaubten Archivar, zwar ungemein belesen, aber ohne relevantes Wissen für die Gegenwart. Diese Sichtweise geht mit der Überzeugung einher, dass alle Phänomene in der Geschichte der Menschen rein kausal zu erklären sind, und es keine historisch begründbaren Hierarchien von Werten gibt. Diese erscheinen vollständig relativiert, umso mehr, als auch Mythos und Religion als massenpsychologische Phänomene »erklärt« werden und somit »entzaubert« sind. Dieses Problem beschäftigt im letzten Drittel des 19. Jahrhunderts alle sich historisch begründenden Disziplinen, von der Rechtswissenschaft über die Religion und Religionswissenschaft, die allgemeine Geschichte bis hin zur Kunst- und Architekturgeschichte. Markant hat dies Friedrich Nietzsche in seiner Zweiten Unzeitgemäßen Betrachtung, Vom Nutzen und Nachtheil der Historie für das Leben 1874 beschrieben. ${ }^{6}$ Der neue Typus des Historikergelehrten sei zu einem Eunuchen geworden, der sein Gegenüber, die Geschichte, nur verehren, mit ihr aber keine Kinder für die Zukunft zeugen könne. Als Auswege aus dem Dilemma stellt Nietzsche neben die hier verspottete antiquarische Geschichtsschreibung eine »kritische $\ll, 7$ die aus der Gegenwart heraus das Vergangene beurteilt und bewertet, vor allem aber eine »monumentalische« Geschichtsschreibung, die - die Weltläufe vereinfachend und

Steinhauser, Monika (Hg.): "Geschichte allein ist zeitgemäß". Historismus in Deutschland, Lahn-Giessen: Anabas 1978. Im Buch ist in grundlegender Weise und im Anschluss an Reinhardt Koselleck die Problematik von Geschichtsschreibung und Architektur behandelt.

6 | Vgl. Nietzsche, Friedrich: "Unzeitgemäße Betrachtungen, zweites Stück: Vom Nutzen und Nachtheil der Historie für das Leben " [1874], in: Ders., Werke. Kritische Gesamtausgabe. 3. Abteilung, Bd. 1: Die Geburt der Tragödie. Unzeitgemäße Betrachtungen I-III (1872-1874), hg. v. Giorgio Colli u. Mazzino Montinari, Berlin/ New York: De Gruyter 1972, S. 239-330.

7 | Ebd., S. 265. 
heroisierend - dem Großen dazu dienen soll, noch größer zu werden und somit unmittelbar auf die Gegenwart zu wirken. Später, etwa bei Max Weber, wird sich die Erkenntnis durchsetzen, dass auch die antiquarische Geschichtsschreibung niemals so neutral und interesselos ist, wie dies lange ihr Anspruch war, sondern immer von einem aktuellen und vital erlebten Heute ihren Blick in die Vergangenheit richtet. ${ }^{8}$

Die um 1900 virulente Krise des Historismus macht die Konstruiertheit von Geschichtsauffassung und Geschichtsschreibung nachdrücklich bewusst. Wenn aber das Vertrauen in eine ehedem als objektiv gegebene und belastbare große historische Erzählung verloren gegangen ist, so muss dies auch für die auf diesem Paradigma aufbauende historistische Architektur gelten.

Maurras' Auffassung, die von zahlreichen Architekten seiner Zeit geteilt wird, ist als eine Spielart monumentaler Geschichtsschreibung zu sehen: Sie soll ein ewig wirkendes, dynamisch verstandenes Prinzip, dasjenige des »klassischen Geistes«, 9 als gegenwärtige Heilsbotschaft darlegen. Sie stellt sich damit gegen die schon zeitgenössisch oft kritisierte antiquarische Haltung, die in der historistischen Architektur manifestiert ist, in der historische Stile zwar oberflächlich gewusst und präzise imitiert werden, ohne dass dabei aber ihre Relevanz für die Gegenwart offengelegt würde. Es ist ein geläufiger Topos der damaligen Architekturkritik, dass die detailgetreue Formenimitation keinerlei gegenwartsbezogene Sinnstiftung leiste, und insofern unverantwortlich und lügnerisch sei.

Doch es gibt nicht nur eine Vielfalt an verschiedenen Geschichtsauffassungen. Die unterschiedlichen Verständnisweisen von Geschichte schreiben sich auch in jede einzelne ersonnene oder auch gebaute Architektur ein beziehungsweise werden durch sie mitgestaltet. Denn die Referenz auf Vergangenes in der Architektur setzt voraus, dass die Architekten zumindest implizit über Modelle verfügen, nach denen Geschichte $z u$ beschreiben ist. Aus diesem Grund handelt es sich bei meinen Ausführungen, wie der Titel etwas kalauernd andeutet, nicht

8| Vgl. Wittkau-Horgby, Annette: Historismus. Zur Geschichte des Begriffs und des Problems, Göttingen: Vandenhoeck \& Ruprecht 1994; Oexle, Otto Gerhard: Geschichtswissenschaft im Zeichen des Historismus, Göttingen: Vandenhoeck \& Ruprecht 1996; Oexle, Otto Gerhard/Rüsen, Jörn (Hg.): Historismus in den Kulturwissenschaften, Köln/Weimar/Wien: Böhlau 1996.

9| Ch. Maurras: Anthinea, S. 171. 
um eine "Geschichte der Architektur« oder »Architekturgeschichte«, sondern um eine Art »Geschichte der Architektur der Geschichte« - den Versuch einer Historisierung von Geschichtsauffassungen innerhalb der Architekturgeschichte. Diese Historisierung soll auch als kritischer Kommentar zu den Traditionen unserer Disziplin verstanden werden, die - aus dem Geist des eben angedeuteten antiquarischen Historismus im 19. Jahrhundert geboren - weiterhin vielfach primär formalistisch verfahren, Formenvorbilder oder Formenvergleiche, sogenannte »Kontexte« und vorgängige Leitideen erkunden möchten, ohne zu bedenken, welche Geschichts- und Zeitvorstellungen eigentlich jeweils mit derartigen Bezügen verbunden sind oder über sie aktiviert werden sollen. ${ }^{10}$

\section{Die Gegenwart der Vergangenheit}

Die spezifischen Auffassungen von Geschichte zeigen sich bereits im Mittelalter, also in einer Zeit, in der gar kein Konzept von Geschichte beziehungsweise keine Historiographie im modernen Sinne eines von Menschen bewirkten, von Zufällen ebenso wie von Kausalität geprägten und von Menschen dargestellten Gesamtkontinuums existiert. Gleichwohl spielt Geschichtsbewusstsein eine eminente Rolle, da die irdische Zeit als Teil des göttlichen Schöpfungswerks zu verstehen war und ihre interpretierende Beschreibung als Aufdeckung der Offenbarung, der providentia, zu gelten hatte. Insbesondere, wenn die Narration typologisch argumentiert, also Geschehnisse auf vorbildhafte biblische oder mythische Ereignisse beziehen kann, zeigt sich göttliche Vorsehung in Bezug auf die irdische Geschichte. Insofern beruht mittelalterliches Geschichtsbewusstsein nicht auf Alterität zur Gegenwart sondern auf der beständigen $\gg$ Gegenwart der Vergangenheit« ${ }^{11}$ In ihrem selektiven

10 | Vgl. Rüsen, Jörn: Historik. Theorie der Geschichtswissenschaft, Köln: Böhlau 2013; Koselleck, Reinhart/Lutz, Heinrich/Rüsen, Jörn (Hg.): Formen der Geschichtsschreibung, München: dtv 1982; Koselleck, Reinhart: "Geschichte", in: Otto Brunner/Werner Conze/Reinhart Koselleck (Hg.), Geschichtliche Grundbegriffe. Historisches Lexikon zur politisch-sozialen Sprache in Deutschland, Stuttgart: Klett 1975, Bd. 2, S. 593-717.

11 | Goetz, Hans-Werner: Geschichtsschreibung und Geschichtsbewußtsein im hohen Mittelalter, Berlin: Akademie Verlag 1999, S. 424. Vgl. Schmale, Franz- 
Charakter ist Geschichtsschreibung im Mittelalter häufig auf Institutionen wie Fürstentümer, Klöster und seit dem späten Mittelalter auch Städte bezogen, deren Anziennität die Stellung der jeweiligen Institution im Heilsplan legitimieren konnte.

Auf das architektonische Geschehen bezogen wird so verständlich, dass es eine Reihe programmatischer Bezugnahmen auf ältere Bauten, beispielsweise auf die Jerusalemer Grabeskirche, St. Peter in Rom, die Aachener Pfalzkapelle Karls des Großen oder später, ab dem 13. Jahrhundert, auf berühmte Pariser Architekturen wie etwa die Sainte Chapelle geben konnte. Doch sind solche Referenzen nicht als Anerkennung historischer Epochen, sondern als Nachfolge eines heilsgeschichtlichen exemplum zu verstehen. Zumeist geht es dabei auch nicht primär um die Wiederholung einer architektonischen Form; vielmehr ist diese das Ergebnis einer planvollen Bauinitiative des Bauherren, mit der dieser sich auf eine lobenswerte vorbildliche Person bezieht. ${ }^{12}$

Auch ein in ein Bauwerk eingeschriebener dezidierter zeitlicher Rückbezug ist als Veranschaulichung von ununterbrochener Kontinuität zu verstehen. Darstellen lässt sich dies etwa für die Kathedrale von Reims (Abb. 2). Der berühmte gotische Neubau aus den Jahren 1207 bis circa 1280 argumentiert in seinem Bildprogramm scheinbar stark »historisch «. Die herausragende Stellung der Metropolitankirche innerhalb seiner Suffragane, der direkte Rombezug sowie das Prärogativ des Reimser Erzbischof, die Salbung und damit Sakralisierung des französischen Monarchen vorzunehmen, und schließlich die kontinuierliche Genealogie der französischen Herrscher sind teilweise redundant ausformuliert. Dabei schwingt eine ekklesiologische Deutung mit, die die Spende des Sakraments der Salbung eng an die Reimser Metropolitankirche bindet. Diese Topoi entstammen insbesondere der von dem Kanoniker Flodoardus in der Mitte des 10. Jahrhunderts verfassten Historia Remensis Ecclesiae. ${ }^{13}$

Josef: Funktion und Formen mittelalterlicher Geschichtsschreibung. Eine Einführung, Darmstadt: Wissenschaftliche Buchgesellschaft 1993.

12 | Vgl. Freigang, Christian: "Imitatio in Gothic Architecture: Forms versus Procedures", in: Zoë Opacic/Achim Timmermann (Hg.), Architecture, Liturgy and Identity: Liber Amicorum Paul Crossley, Turnhout: Brepols 2011, S. 297-313.

13 | Vgl. Flodoardus Remensis: Historia Remensis Ecclesiae, hg. v. Martina Stratmann, Hannover: Hahn 1998, sowie Sot, Michel: Un historien et son église au Xe siècle: Flodoard de Reims, Paris: Fayard 1993. 
Abb. 2, Kathedrale von Reims, Westfassade, zweite Hälfte 13. Jahrhundert.

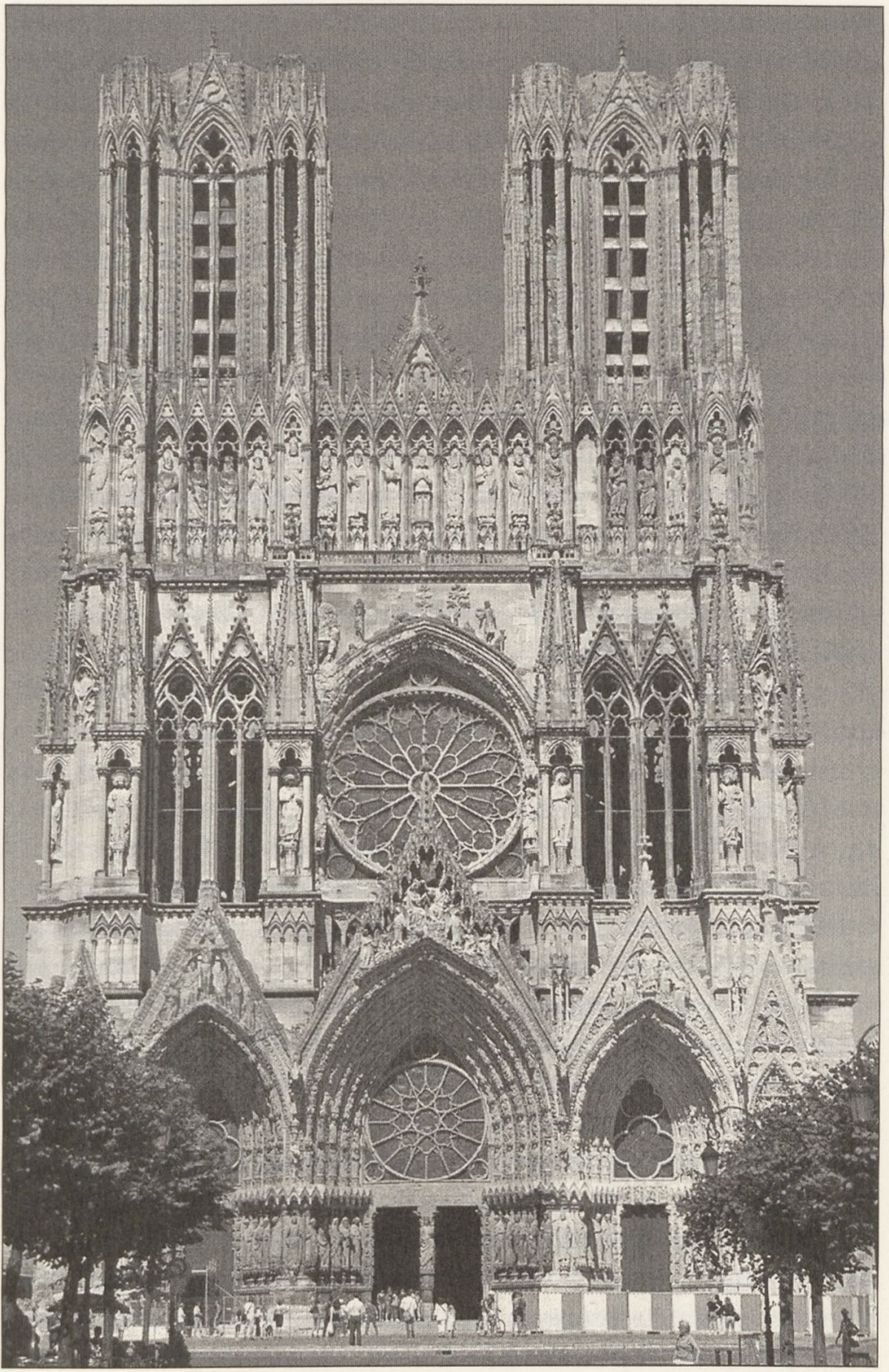

Quelle: http://commons.wikimedia.org/wiki/File:Reims_Kathedrale. jpg\#mediaviewer/File:Reims_Kathedrale.jpg vom 30. März 2015. 
Die Kathedrale vollendet damit den christlichen Heilsplan, indem sie gleichsam an vorderster Front die apostolische Missionierung der Menschheit betreibt und diese eng auf das französische Königtum bezieht. Mehr noch: Die vorbildhaften Erzbischöfe der Reimser Kirche, Nicasius im 4. Jahrhundert sowie Ebbo und Hinkmar im 9. Jahrhundert, agieren auch als die wesentlichen architektonischen Erbauer der Kirche. Der Neubau fügt sich in diese historische Logik ein, die dank der Zyklen der Reimser Bischöfe und der fränkischen Könige als eine genealogisch kontinuierliche ausgewiesen ist. ${ }^{14}$ Als Gründerbischof des Neubaus erscheint nunmehr aber, im Glasmalereizyklus des Obergadens des gotischen Neubaus, Henri de Braine (de Dreux, reg. 1227-1240) inmitten eines Apostelkollegiums sowie der Verbildlichungen der Suffraganbischöfe stehend. ${ }^{15}$ Die historische Dimension erscheint also als eine sich zeitlich immer wieder bestätigende Konstellation. Und somit entspricht es auch dieser Erfüllung des Heilsplans, das Bauwerk, das heißt die Kirche, nach den neuesten Techniken und Formen als Ausdruck höchster Pracht und Festlichkeit zu errichten, die solchermaßen auch die historische Suprematie ausdrückt. Geschichtlichkeit ist insoweit enthalten und ausgedrückt, als sie in der Gegenwärtigkeit des materiellen Gebäudes aufgeht, keinesfalls aber als deren Anderes, Fremdes oder Vergangenes ausgewiesen ist.

Anders stellt sich dieses Verhältnis zur Geschichte seit dem 15. Jahrhundert dar. Die Konkurrenz der italienischen Stadtstaaten, aber auch der Ausbau Roms als Zentrum des Christentums befördern nun den Drang, Altehrwürdigkeit und daraus ableitbare Rechte durch einen vielfältigen Bezug auf die Antike zu untermauern. Zu dieser Strategie gehören auch die sogenannte Wiederentdeckung der antik-römischen Baukunst, ihr detailliertes Studium und ihre nun machtvoll einsetzende architekturtheoretische Bewertung. ${ }^{16}$ Was hier beginnt und dem Mittelalter praktisch fremd war, ist die Konzeption von Architektur in ihrer

14 | Vgl. Lillich, Meredith Parsons: "King Solomon in Bed, Archbishop Hincmar, the ,Ordo of 1250, and the Stained-Glass Program of the Nave of Reims Cathedralı, in: Speculum 80 (2005), S. 764-801.

15 | Vgl. Kurmann, Peter: „Französische Bischöfe als Auftraggeber und Stifter von Glasmalereien. Das Kunstwerk als Geschichtsquelle", in: Zeitschrift für Kunstgeschichte 60 (1997), S. 429-450.

16 | Vgl. Günther, Hubertus: Das Studium der antiken Architektur in den Zeichnungen der Hochrenaissance, Tübingen: Wasmuth 1988. 
bildlichen Erscheinung, unabhängig von der Qualität der Techniken und Materialien. Die richtigen Proportionen und Details einiger Schlüsselelemente wie Säulen und Gebälke steigen nunmehr zu einer dank des Bilddrucks rasch kanonisierten Bildersprache der Weltarchitektur auf. ${ }^{17}$ Die Trias »dorisch, ionisch, korinthisch « etwa dominiert weltweit bis in das 19. Jahrhundert das anspruchsvolle Bauen in europäischer Tradition. Im 15. Jahrhundert ist dies aus einer neuen Form von Geschichtsschreibung legitimiert, die die Historie als Handlungsanleitung für die Gegenwart versteht. Die explizit als renovatio benannte Erneuerung Roms versteht sich als gleichsam familiäre Pflege der Elterngeneration. ${ }^{18}$ Rom beziehungsweise die Antike gilt als »hinfällige Mutter«, die nunmehr zu verjüngen und zu beleben ist. ${ }^{19}$ Solches lesen wir schon bei Hildebert von Lavardin oder bei Petrarca, dann bei Flavio Biondo. ${ }^{20}$ Die augustinische Zweiteilung der Zeitenverläufe in eine himmlisch-überzeitliche und eine untergeordnete irdisch-zeitliche Geschichte beziehungsweise die Einteilung in vier in das römische Reich mündende Weltzeitalter weicht einer neuen Auffassung, der Profangeschichte. Diese versteht sich als unparteiische Beschreibung objektiver Begebenheiten, innerhalb derer der Antike eine unhintergehbare Größe und Integrität zu bescheinigen ist. Die Dreiteilung in Altertum, Mittelalter und Neuzeit fasst die letzte Epoche als Gegenwart, die ihre Verfasstheit aus dem Studium der Antike ableitet. Insofern gehören die neuen Renaissancebauten im Verständnis der Zeit gleichsam der Antike an, sie sind Ausdruck einer bußfertigen Überwindung der barbarischen Zeit des Mittelalters, nach der nunmehr Gesundung und Verjüngung eintreten kann. Es ist dieser Geschichtsauffassung zu verdanken, dass Leon Battista Alberti eine Art christlichen Triumphbogen als Kirchenfassade in Rimini errichten kann (Abb. 3), oder Donato Bramante einen christianisierten antiken Rundtempel zu Ehren

17 | Vgl. Carpo, Mario: Architecture in the Age of Printing: Orality, Writing, Typography, and Printed Images in the History of Architectural Theory, Cambridge/ London: MIT Press 2001.

18 | Vgl. Muhlack, Ulrich: Geschichtswissenschaft im Humanismus und in der Aufklärung. Die Vorgeschichte des Historismus, München: Beck 1991, S. 164-172.

19 | Heckscher, Wilhelm Sebastian: Die Romruinen. Die geistigen Voraussetzungen ihrer Wertung im Mittelalter und in der Renaissance, Würzburg: Mayr 1936 , S. 12 .

20 | Vgl. ebd., S. 17-37. 
der Kreuzigungsstätte des heiligen Petrus. Die Zeit zwischen Altertum und Gegenwart wird gleichsam kontrahiert, die Distanz zwischen ihnen aufgehoben. Aus solchen Gründen sind auch in der Traktatliteratur vielfach, etwa bei Serlio oder Palladio, antike und moderne Monumente in einem Zug abgebildet und besprochen..$^{21}$

Abb. 3, Leon Battista Alberti: San Francesco oder Tempio Malatestiano, Rimini um 1450.

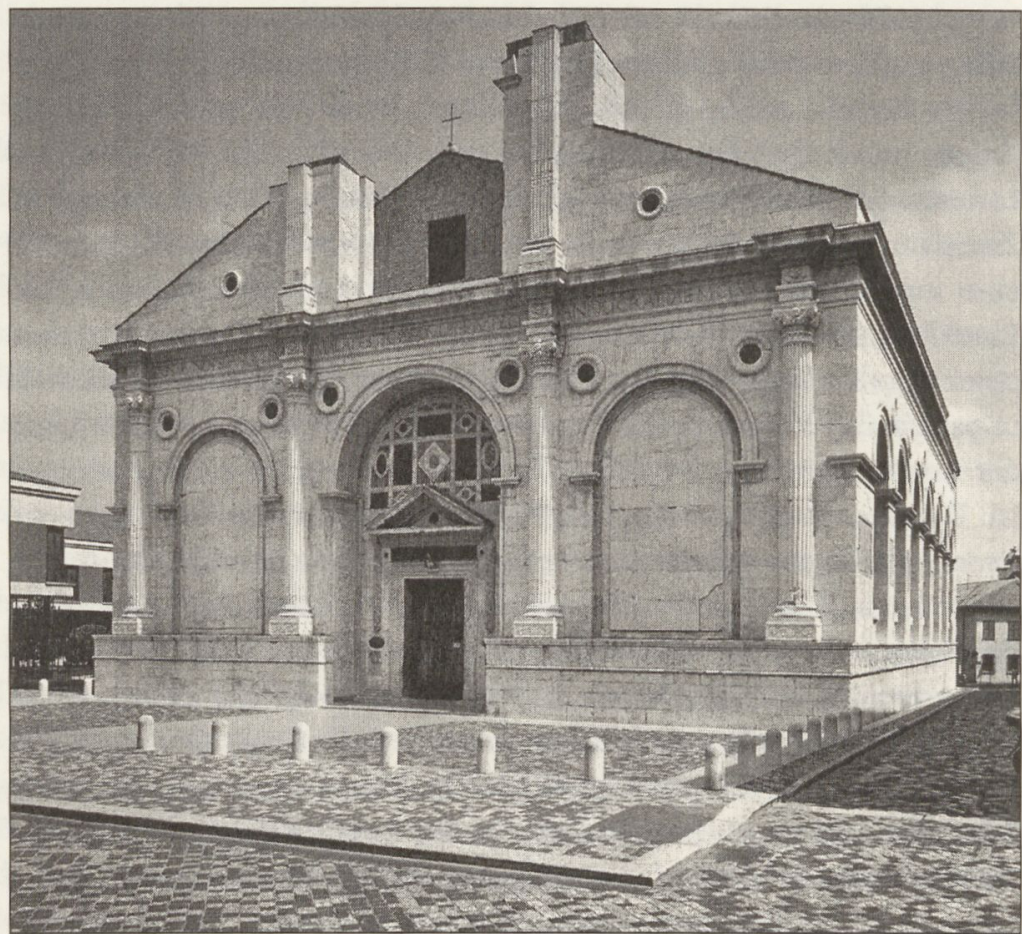

Quelle: Bulgarelli, Massimo, et al. (Hg.): Leon Battista Alberti e l'architettura, Mailand: Silvana Ed. 2006, S. 284.

21 | Vgl. Serlio, Sebastiano: Regole generali di architettura sopra le cinque maniere degli edifici, cio e thoscano, dorico, ionico, corinthio et composito, con gli essempi dell'antiquita, che, per la magior parte concordano con la dottrina di Vitruvio, Venedig: Marcolini da Forli 1537; Palladio, Andrea: I quattro libri dell'architettura, hg. v. Licisco Magagnato u. Paola Marini, Mailand: II Polifilo 1980 [1570]; Krufft, Hanno Walter: Geschichte der Architekturtheorie, München: Beck 1985. 
Zum Problem wird diese Kontraktion der Architekturgeschichte im Laufe des 16. Jahrhunderts, als das Wissen um die Vielfalt der historischen Architekturen vielschichtiger wird und eine systematische Einordnung in eine Entwicklung, in eine historisch fundierte Chronologie, erfordert. Grundsätzlich divergieren seit der Zeit um 1600 zwei Geschichtsauffassungen. Die angestammte Parallelisierung von Menschheits- und Architekturgeschichte, beides beginnend mit den Stammeltern beziehungsweise der bescheidenen Urhütte und beides in der Antike zu idealer Perfektion geführt, wird in der Querelle des Anciens et des Modernes um 1700 erkenntnistheoretisch problematisiert. Die Frage, ob das Jahrhundert Ludwigs XIV. die antike Perfektion erreicht habe oder sie gar übertreffe, führt nicht nur zur Relativierung von Geschmacksurteilen, sondern auch zur Erkenntnis von grundsätzlich evolutiven, aber nicht geradlinigen und zudem vielfältigen historischen Kontinuen - etwa in der Naturwissenschaft oder in den Künsten - innerhalb der Weltverläufe, die einzig durch raisonnement zu systematisieren seien. ${ }^{22}$ Dem gegenüber tritt eine gleichsam deszendierende Auffassung: Gott als oberster Architekt habe in der Superarchitektur des Jerusalemer Tempels eine nicht zu überbietende Urarchitektur geschaffen, von der sich das nachfolgende Bauen ableiten ließe. Dessen Entwicklung, die etwa bei Nicolaus Goldmann und Christoph Leonhard Sturm um 1700 ausschweifend beschrieben ist, folge einer göttlich bestimmten historischen Abfolge, die sich insbesondere im Nacheinander der fünf Säulenordnungen toskanisch, dorisch, ionisch, korinthisch und komposit - nachweisen lasse. Anhand kleinteiligster, haarspalterischer Argumente kann Sturm eine perfekte mathematische wie evolutionäre Ordnung nachweisen, die letztendlich Ausdruck einer göttlich vorbestimmten Weltgeschichte ist. Die Säulenordnungen werden bei ihm vermittels einer Reihe axiomatischer Setzungen so sehr zur Matrix der Weltgeschichte, dass es möglich erscheint, eine sechste Säulenordnung zu deduzieren, die neben den italienschen und griechischen Ordnungen nun auch dem protestantischen »Teutschen Volke« den ihm zukommenden historischen Platz einräumen soll. Die durch göttliches Wirken mathematisch perfekte und in allen Details konstruierte Weltmechanik lasse sich, da sie grundsätzlich einer

22 | Vgl. Perrault, Charles: Parallèle des anciens et des modernes en ce qui regarde les arts et les sciences, eingeleitet von Hans Robert Jauss, München: Eidos 1964. 
naturwissenschaftlichen Gesetzlichkeit folgt, extrapolierend ergänzen beziehungsweise rekonstruieren. Die Säulenordnungen seien eindeutige historische Indikatoren, die - natürlich zeigt sich hier ein Zirkelschluss die harmonische Ordnung der göttlichen Schöpfung und ihrer Entwicklung erweisen sollen. ${ }^{23}$

\section{Historische StiLe}

Eine entscheidende Wende ereignet sich in der Mitte des 18. Jahrhunderts, als sich der oben umrissene moderne Geschichtsbegriff herausbildet, der sich alsbald in die Idee einer Universalgeschichte umsetzt, die die Welt als komplex kausal vernetztes und zeitlich dynamisches Gebilde zu beschreiben sucht. In bezeichnender Weise haben dabei auch Kunst und Architektur ihren Anteil, der insbesondere durch Winckelmann in der ersten Universalgeschichte der Kunst formuliert wurde. ${ }^{24}$ Das führt aber nicht, wie bisweilen zu lesen, zur Entwertung ästhetischer Kanones, sondern vielmehr zu einer neuen semantischen Aufladbarkeit von historischen Stilen. Sie gelten nunmehr als Resultate und Metonymien komplexer historischer Konstellationen. Vor allem aber wird in ihnen die grundsätzliche Trennung von historischem Objekt und interpretierendem Subjekt deutlich, die im Zuge der modernen Geschichtsschreibung aktiviert wurde. Das bildet den Hintergrund für die spezifische Debattenkultur des architektonischen Historismus mit ihren zahlreichen Wettbewerben, Konkurrenzentwürfen und der einsetzenden Architekturkritik und -publizistik. Jedwede Architektur ist nunmehr in der Geschichte zu verorten, in der sie als das Resultat komplizierter, aber bedeutungsvoller und politisch zu verstehender Zusammenhänge erscheint.

Als Beispiel für diese neue Sichtweise soll die Umgestaltung Münchens, als relativ neuer königlicher Kapitale unter Ludwig I., zu

23 | Vgl. Freigang, Christian: "Göttliche Ordnung und nationale Zeitgemäßheit: Die Querelle des Anciens et des Modernes in der deutschen Architekturtheorie um 1700 ", in: Sylvia Heudecker/Dirk Niefanger/Jörg Wesche (Hg.), Kulturelle Orientierung um 1700. Traditionen, Programme, konzeptionelle Vielfalt, Tübingen: Niemeyer 2004, S. 122-142.

24 | Vgl. Winckelmann, Johann Joachim: Geschichte der Kunst des Altertums, Dresden: Walther'sche Hofbuchhandlung 1764. 
einem wahren Geschichts- beziehungsweise Architekturgeschichtsmuseum dienen. Der Monarch lässt nicht nur drei programmatische Museen als Präsentationsorte der Geschichte der Kunst errichten, die Glyptothek für die Antiken, die Alte Pinakothek für die älteren Gemälde, die Neue Pinakothek für die jüngeren Schulen. Zahlreiche historistische Bauten bestimmen darüber hinaus die umfassenden Stadterweiterungen unter Ludwig, darunter der sogenannte Königsbau der Residenz, der repräsentative Wohnflügel des Monarchen, der sich mit seiner Neorenaissancefassade plakativ der Altstadt Münchens zuwendet (Abb. 4). Mit der Anspielung auf den Palazzo Pitti in Florenz soll sich das Mäzenatentum der Medici mit dem fördernden Wirken des bayrischen Königs verbinden.

Die Hofkapelle folgt hingegen süditalienischen Modellen, eine Erinnerung an den Grand Tour des gebildeten Königs und zugleich an die Glanzzeit deutscher Herrscher in Italien. Der Festsaaltrakt im Norden paraphrasiert palladianische Modelle als Rahmen gediegener Festlichkeit. Das Siegestor am Ende der neu angelegten Ludwigsstraße rekurriert nun sowohl auf römische Triumphbögen auf dem Forum romanum wie auf ihre Reflexe in dem Pariser Arc du Caroussel. Das Ensemble um den Königsplatz mit den Propyläen und der Glyptothek wiederum resultiert aus genauen archäologischen Studien des Architekten Leo von Klenze und beansprucht insgesamt, einen Ort höchster Zivilisation als angemessenen Rahmen für die verehrten Antiken zu evozieren. Man könnte die Aufzählung mit anderen Monumenten fortsetzen, der Feldherrnhalle, der Ludwigskirche und so weiter. Insgesamt ergibt sich eine eigenartige Gemengelage unterschiedlicher historischer Referenzen mit vielfältigsten Assoziationen. Anhand der Münchener Architekturen des frühen 19. Jahrhunderts wäre gleichsam eine Geschichte der Welt zu erzählen, eine Geschichte, die keine herausgehobene Epoche betont, sondern Vielfalt kennt: attische Demokratie, römisches Weltreich, deutsch-italisches Kaisertum, florentinische Kommune, barockes Italien (in Form der schon älteren Theatinerkirche), aufgeklärtes England, Napoleon und die Befreiungskriege. Hinzu kommen in den bildlichen Ausstattungsprogrammen die bayrische Landesgeschichte sowie der Nibelungenstoff. ${ }^{25}$

25 | Vgl. Romantik und Restauration. Architektur in Bayern zur Zeit Ludwigs I. 1825-1848. Ausstellung der Architektursammlung der Technischen Universität München und des Münchner Stadtmuseums in Verbindung mit dem Zentralinstitut 
Abb. 4, Leo von Klenze: Königsbau der Residenz, München 1826-1835.

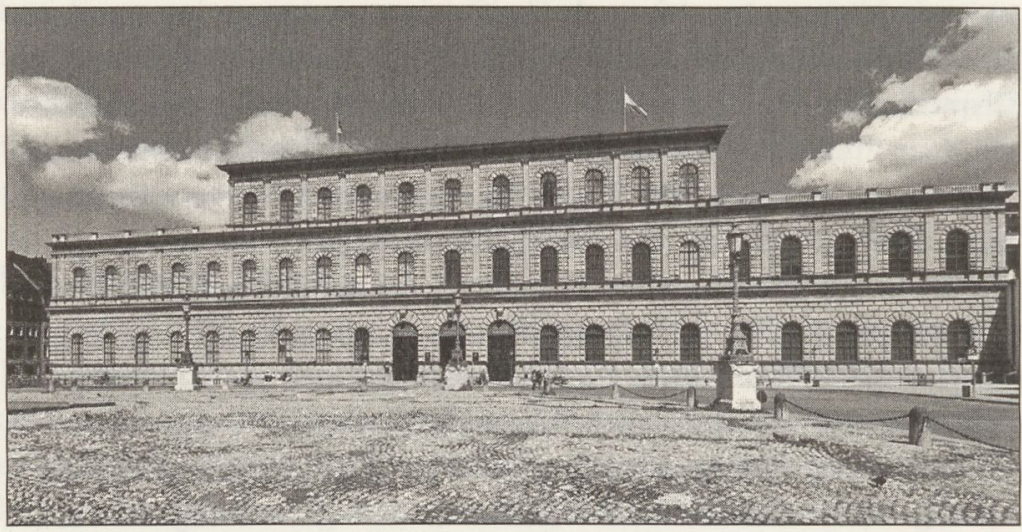

Photographie: Christian Freigang.

Insgesamt ist auffällig, dass all diese Bauten urbanistisch prominent inszeniert sind und in den meisten Fällen öffentlichen Institutionen dienen. Sie rechnen mit der historischen Bildung einer bürgerlichen Öffentlichkeit, um in ihren Aussagen verstanden zu werden, und legitimieren gleichzeitig die Notwendigkeit allgemeiner Bildung. Wenn an der Ludwigstraße die Staatsbibliothek und die Universitätsbauten entstehen, dann zeigt das in signifikanter Weise den Konnex zwischen Geschichtswissenschaft, Bürgertum und historistischer Architektur. Auch wenn die theoretische Geschichtsauffassung Ludwigs beziehungsweise seiner Architekten Leo von Klenze und Friedrich von Gärtner nicht einfach zu definieren ist, so zeugen die von ihnen in Auftrag gegebenen beziehungsweise erbauten Architekturen von der eminent didaktischen Funktion einer universalen Geschichte, die - ganz im Stile der historischen Romane der Zeit - mit unendlich vielen Erzählungen aufzufüllen ist: der tragischen Geschichte des Stauferkaisers Friedrich II., dem Aufstieg und Fall der Medici-Familie oder den bedrohlichen Eroberungszügen Napoleons.

Darin liegt aber zugleich der Keim zur Auflösung des Historismus, der auf einer »großen Erzählung« beruht, die eindeutig mit den Bauwerken verbunden werden kann. Sobald sich indes Geschichtserzählungen nicht mehr eindeutig auf Bauwerke projizieren lassen - für die Geschichte des

für Kunstgeschichte [München, Münchner Stadtmuseum, 27. Februar - 24. Mai 1987], hg. v. Winfried Nerdinger, München: Prestel 1987. 
Proletariats gibt es zum Beispiel keine heroischen Vorbildbauten -, sobald Geschichtsschreibung ihre identitätsstiftende Funktion verliert - etwa, weil sie sich als falsch oder irrelevant erweist - und sobald historische Stilreferenzen zu beliebig verwendbaren Klischees werden, muss der Bezug von Architektur auf Geschichte neu geregelt werden. Dies geschieht, wie am Beispiel Maurras' ausgeführt, etwa dadurch, dass strukturelle Relationen zwischen Form und Inhalt eines Gebäudes in eine archetypisch wirksame Überzeitlichkeit - etwa des »griechischen Geistes - überführt werden.

\section{Die Abwicklung der Geschichte}

Man mag einwenden, dass die eingangs dargestellte hymnische Verehrung des Parthenon durch einen herausragenden Vertreter der antiparlamentarischen Fundamentalopposition nichts Besonderes sei, weil ein jeder die griechische Architektur der Antike als Spitzenwerk, damals wie heute, einstufen würde. Aber solche Einstellungen lassen sich nicht einfach als Klischees entwerten. Es geht vielmehr darum, solche Klischees zu kontextualisieren, gerade in Bezug auf differierende Geschichtsauffassungen. Ganz anders als die universalistischen und holistischen Vereinnahmungen des Parthenon geht beispielsweise Le Corbusiers Präsentation des antiken Bauwerks vor. In seiner Epoche machenden Schrift Vers une architecture proklamiert er einen technokratischen Utilitarismus, der insbesondere auf das Wohnen des Menschen fokussiert ist, das - dank neuer technisch-konstruktiver Möglichkeiten sowie rationeller und standardisierter Montagetechniken des Hauses unter Beachtung ökonomischer Parameter - vor allem essenzielle biologische Funktionen zu erfüllen habe. Die ästhetische Befriedigung wird nicht länger sentimental erinnernden Stilformen und Ornamenten überlassen, sondern direkt erfahrbaren, geometrisch gereinigten Formen und Farben sowie dem Faszinosum der Maschine, die absolute Gegenwart erst erlebbar macht. Die "Wohnmaschine« ist insofern ein typisches Produkt dieser Theorie. Von zweckgerichteter Funktionalität, lässt sie sich immer weiter verbessern und erneuern, ungeachtet belastender sentimentaler Erinnerungen an Formen, Traditionen, Geschichte, Nation oder Mythos. In einer berühmten Bildmetapher parallelisiert Le Corbusier in diesem Zusammenhang die Entwicklung des griechischen Tempels, der im Athener Parthenon 
gipfelt (Abb. 5), mit der Veränderung der Formen von Automobilen. Die Evolution der antiken Architektur wird hier explizit als Produktselektion aufgefasst, deren modernes Pendant die faszinierende Maschine - Autos, Flugzeuge, Ozeandampfer und eben auch Häuser - seien beziehungsweise sein sollten. Die Provokation beruht nicht allein auf der respektlosen Kombination von ehrwürdigem Bauwerk und banaler Maschine, sondern ebenso auf der abschätzigen Präsentation der Abbildungen des Athener Parthenon: Diese sind von minderwertigster Druckqualität und vor allem ganz offensichtlich zu Fragmenten zerschnitten, auf verschiedene Seiten verteilt oder, alle graphischen und typographischen Regeln außer Acht lassend, gekippt wiedergegeben.

Abb. 5, Doppelseite mit Parthenon und Propyläen.

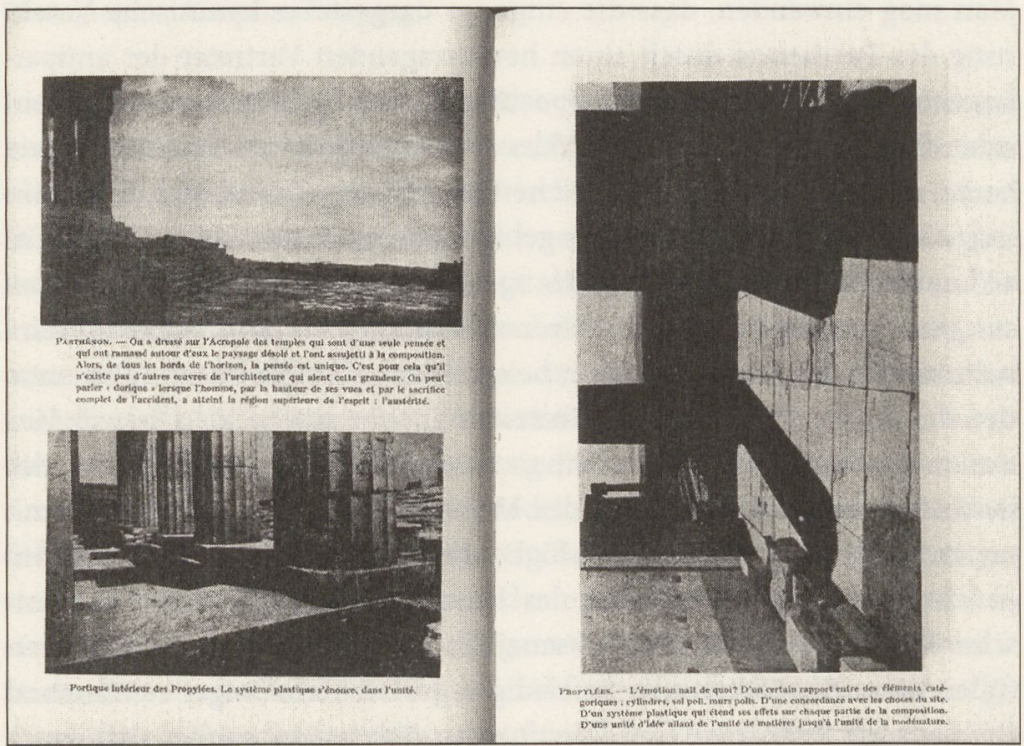

Quelle: Le Corbusier: Vers une architecture, Paris: Ed. Crès 1924, S. 166-167.

Die respektlose Parthenonrezeption als Produktselektion - und nicht als historische Referenz/Reverenz - geht mit einer völlig fragmentierten Geschichtsdarstellung einher, die sich nicht nur hier zeigt, sondern das Gesamtwerk von Le Corbusier durchzieht. Vers une architecture rekurriert immerhin auf die griechische und römische Antike, das islamische Bauen, Altpersien und Michelangelo, unterschlägt aber die gesamte Tradition 
des europäischen - und insbesondere des französischen - Bauens, um dann paukenschlagartig eigene Villenbauten als kompletten Neubeginn der Architektur zu proklamieren. Tradition und Kontinuität sind nurmehr in offensichtlichen Klischees oder respektlosen Pervertierungen präsent, die Lücken zwischen den Epochen sind riesig, die Intonierung eines kompletten Neuanfangs in Form von vergleichsweise bescheidenen Wohnbauten eine groteske Maßstabslosigkeit. Einige Grundtheoreme der älteren Geschichtswissenschaft sind dabei zwar überdeutlich präsent, wie das Lernen aus modellhaften Lösungen, aber zugleich werden in provozierender Eitelkeit neue Akzente gesetzt: Produktselektion statt vollendete Harmonie gemäß dem historisch kontinuierlichen Prinzip der imitatio naturae.

Die Abwicklung der Geschichte ist hier also vollständig im Gange. Der Versuch, aus dem historistischen Paradigma zu entkommen, wird generell zum Kennzeichen der Moderne, die unter anderen bei Walter Gropius, Ludwig Hilberseimer, Hannes Meyer und Ernst May vorwiegend anthropologisch, unter Bezug auf angebliche Grundbedürfnisse des Menschen, argumentiert und konzipiert. Diese Auffassungen kollidieren allerdings in bezeichnender Weise mit den Vorstellungen einer Weltarchitektur, wie sie im Programm für den Völkerbundpalast an den Ufern des Genfer Sees von 1926 wirksam wurde. Als Resultat des Wettbewerbs errichtete von 1932 bis 1937 ein internationales Architektenteam um Paul Nénot und Julien Flegenheimer innerhalb eines arkadischen Parkgrundstückes ein monumentales neoklassizistisches Gebäude, das den Anspruch erhob, das harmonische Zusammenwirken der Völker der Erde architektonisch auszudrücken (Abb. 6).

Der monumentale Klassizismus, dessen sich die Architekten bedienten, wurde in der zeitgenössischen Diskussion zwar einerseits als eine Art synthetisierende Weltarchitektur präsentiert, die die Typologien einer starken Festung, eines Tempels von lyrischer Anmut und eines staatlichen Palasts vereine. Andererseits wurden dabei die griechisch-mediterranen Referenzen besonders herausgestellt. Klassizismus, überhistorische Kontinuität und mittelmeerische Kultur formulieren eine in die Zukunft fortschreibbare longue durée: keine Geschichte, sondern das Axiom einer ewig sich perfektionierenden Zivilisation. Noch 30 Jahre zuvor hätte man für eine vergleichbare Bauaufgabe wohl eher ein Super-Pasticcio entworfen. Konträr zu Nénot und Flegenheimer versteht sich der ebenfalls prämierte, aber nicht weiter verfolgte Entwurf von Le Corbusier. Colla- 
geartig sind einige architekturhistorische Verweise gegeben: barocke Flügelbauten, ein skulpturales Denkmal am Haupteingang. Vor allem aber werden vielfältige anthropologische und funktionalistische Faktoren ins Feld geführt, um eben dadurch dem Anspruch einer übernationalen, von allen divergierenden regionalen Traditionen befreiten Architektur gerecht zu werden.

Abb. 6, Julien Flegenheimer und Paul Nénot: Verwaltungsgebäude der UNO (ehemaliger Völkerbundpalast), Genf 1927-1937.

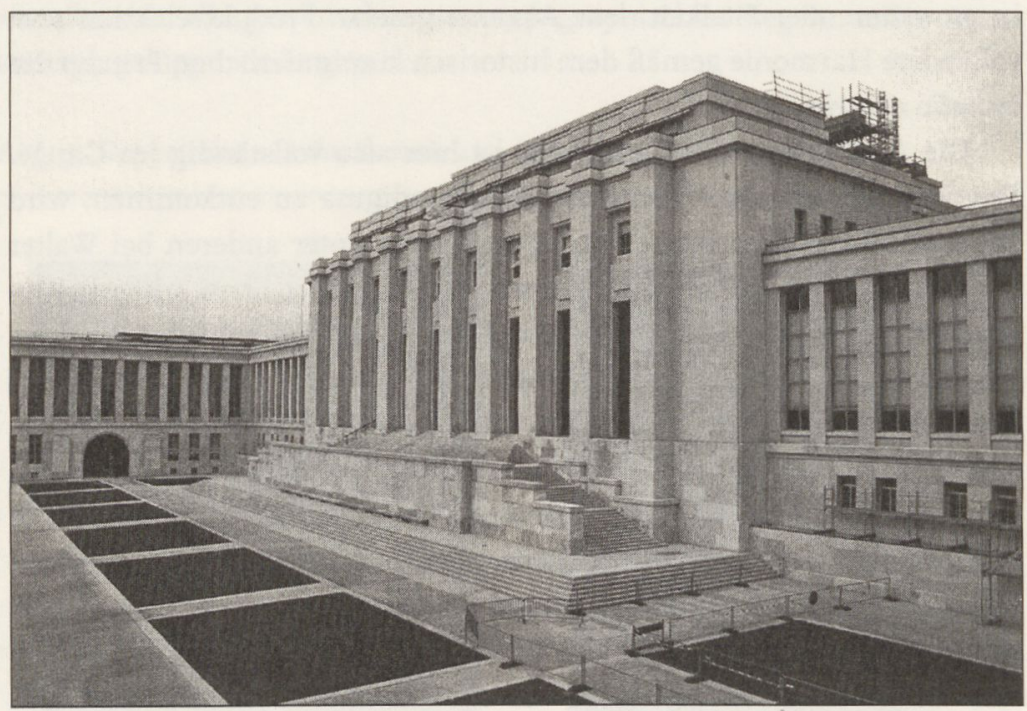

Quelle: Archiv Christian Freigang.

Die im Zusammenhang des Völkerbundpalastes ausgetragenen Diskurse sind durchaus Teil des Historismusdilemmas und können auf dessen Lösungsversuch durch Max Weber bezogen werden. ${ }^{26}$ Dieser Versuch beruht darauf, historisches Erkennen und werteorientiertes Handeln voneinander zu trennen beziehungsweise in einer Wechselwirkung zu begreifen. Erkennen ist insofern nur für begrenzte Bereiche der Vergangenheit möglich, historische Erkenntnis kann für sich also ihre Relevanz für das

26 | Vgl. Weber, Max: "Die ,Objektivität, sozialwissenschaftlicher und sozialpolitischer Erkenntnis“ [1904], in: Ders., Gesammelte Aufsätze zur Wissenschaftslehre, hg. v. Johannes Winckelmann, Tübingen: Mohr-Siebeck 1973, S. 146-214. 
Heute gar nicht begründen, sondern es obliegt anderen Bereichen, zum Beispiel Religion und Ethik, Normen zu setzen, denen sich ein Kollektiv unabhängig von wissenschaftlichen Begründungen unterwirft. Diese Akzeptanz allerdings geschieht durch die wertorientierte Konstruktion von idealtypischen Begriffen in der Geschichte. Letztendlich ist also historische Erkenntnis dauernde Spiegelung des eigenen aktuellen Handelns in der Gegenwart. Eben solche Muster lassen sich bei Le Corbusier und anderen modernen Entwürfen nachverfolgen: Die Herauslösung aus dem historischen Paradigma antwortet auf die autoritäre, gleichwohl vergebliche Normsetzung, wie sie das penetrant klassizistisch ausgeführte Gebäude vorgibt, und fordert stattdessen die Unterwerfung unter anthropologisch begründete Normen. Absolut ist die geschichtsvergessende Moderne dennoch nicht, denn sie funktioniert nur in ihrer provokativen Absetzung vom historistischen Paradigma, das als Hintergrundfolie immer erkennbar bleiben muss, um als sentimental, ungesund, verlogen und irrelevant geächtet und somit auf Distanz gehalten zu werden. Von diesem historistischen Paradigma wird die Moderne außerdem umgehend wieder eingeholt: Le Corbusier und seinesgleichen sind natürlich sehr schnell Geschichte geworden, mit eigenen Erzählungen, Mythen, Traditionen und Symbolen.

\section{Zeitgenössische EntwickLungen}

Die Bedeutung von Geschichtskonstruktionen lässt sich auch für die Gegenwart trefflich veranschaulichen. Signature buildings und Themenparks in den global cities bedienen sich häufig bildgewordener Stereotypen etwa von Venedig oder Paris - aus dem Reservoir einer populären Weltgeschichte. Wenn in Deutschland der Architekt Christoph Mäckler an vielen Orten neue Hochhausbauten - etwa den Frankfurter Opernturm oder das Zoofenster in Berlin - errichtet, so kann er sich der positiven Kommentare fast sicher sein. Dies gilt vor allem deshalb, weil es sich um renditebewusste Investorenarchitektur handelt, die zugleich eine nachhaltige Stadtreparatur verspricht. Mäcklers Bauten nehmen beispielsweise auf das alte Konzept der Korridorstraße Rücksicht, auf Blickachsen und Proportionen, legen Wert auf ein gediegenes Äußeres und vor allem auf historische Anbindungen insbesondere an die Wolkenkratzer der zwanziger und dreißiger Jahre in den USA. Für Mäckler werden damit 
dauerhafte und bewährte Kriterien des Städtebaus erfüllt und außerdem denkmalpflegerische Maßnahmen der Stadtsanierung realisiert. Für die Kritiker sind hier Klischees einer als gesund und elegant, zugleich als leistungsstark und solide konnotierten Kulturschicht aktiviert und damit der Rahmen für einen attraktiven Investitionsmix gesetzt, der auf die »gute alte Zeit« der »Roaring Twenties « abhebt. ${ }^{27}$ Auffälligerweise beherbergt der Frankfurter Turm eine Schweizer Bankgesellschaft und eine Kaufhauskette, die sich den Remakes von Haushaltsartikeln der Vorkriegszeit sehr erfolgreich verschrieben hat. In Berlin wird das Gebäude von einem Luxushotel mit alter Tradition und der Nachfolgeeinrichtung des während der Weimarer Republik berühmten Romanischen Cafés genutzt. In solchen Strategien zeigen sich die Unterschiede zu den Geschichtsauffassungen, die beispielsweise in der Münchener Ludwigstraße oder dem Théâtre des Champs Elysées wirksam wurden (Abb. $4+1$ ). Geschichte erscheint bei Mäckler als Fundus von global verfügbaren, entkontextualisierten Images. Zu solchen gehören ebenso das deutsche Altstadt-Fachwerkhaus, das alpenländische Bauernhaus, die überkuppelte Moschee und eben auch der amerikanische Wolkenkratzer: Supersymbole einer Geschichte der Architektur der Geschichte.

\section{LITERATUR}

Borsi, Franco: Die monumentale Ordnung. Architektur in Europa 1929-1939, Stuttgart: Hatje 1987.

Brix, Michael/Steinhauser, Monika (Hg.): »Geschichte allein ist zeitgemäß $\aleph_{\text {. }}$ Historismus in Deutschland, Lahn-Giessen: Anabas 1978.

Bulgarelli, Massimo, et al. (Hg.): Leon Battista Alberti e l'architettura, Mailand: Silvana Ed. 2006.

Carpo, Mario: Architecture in the Age of Printing: Orality, Writing, Typography, and Printed Images in the History of Architectural Theory, Cambridge/London: MIT Press 2001.

27 | Vgl. Dauss, Markus: „Innovative Höchstwerte?? Hochhausbau und -abriss in 'Mainhattan «", in: Christian Freigang/Markus Dauss (Hg.), Das "neue Frankfurt". Innovationen in der Frankfurter Kunst vom Mittelalter bis in die Gegenwart, Frankfurt a.M.: Kramer 2010, S. 124-143. 
Dauss, Markus: »Innovative >Höchstwerte<? Hochhausbau und -abriss in >Mainhattan «, in: Christian Freigang/Markus Dauss (Hg.), Das »neue Frankfurt«. Innovationen in der Frankfurter Kunst vom Mittelalter bis in die Gegenwart, Frankfurt a.M.: Kramer 2010, S. 124-143.

Flodoardus Remensis: Historia Remensis Ecclesiae, hg. v. Martina Stratmann, Hannover: Hahn 1998.

Freigang, Christian: Auguste Perret, die Architekturdebatte und die »Konservative Revolution « in Frankreich 1900-1930, Berlin/München: Deutscher Kunstverlag 2003.

—»Göttliche Ordnung und nationale Zeitgemäßheit: Die Querelle des Anciens et des Modernes in der deutschen Architekturtheorie um 1700«, in: Sylvia Heudecker/Dirk Niefanger/Jörg Wesche (Hg.), Kulturelle Orientierung um 1700. Traditionen, Programme, konzeptionelle Vielfalt, Tübingen: Niemeyer 2004, S. 122-142.

— »Imitatio in Gothic Architecture: Forms versus Procedures«, in: Zoë Opacic/Achim Timmermann (Hg.), Architecture, Liturgy and Identity: Liber Amicorum Paul Crossley, Turnhout: Brepols 2011, S. 297-313.

Goetz, Hans-Werner: Geschichtsschreibung und Geschichtsbewußtsein im hohen Mittelalter, Berlin: Akademie Verlag 1999.

Günther, Hubertus: Das Studium der antiken Architektur in den Zeichnungen der Hochrenaissance, Tübingen: Wasmuth 1988.

Heckscher, Wilhelm Sebastian: Die Romruinen. Die geistigen Voraussetzungen ihrer Wertung im Mittelalter und in der Renaissance, Würzburg: Mayr 1936.

Koselleck, Reinhart/Lutz, Heinrich/Rüsen, Jörn (Hg.): Formen der Geschichtsschreibung, München: dtv 1982.

Koselleck, Reinhart: »Geschichte«, in: Otto Brunner/Werner Conze/ Reinhart Koselleck (Hg.), Geschichtliche Grundbegriffe. Historisches Lexikon zur politisch-sozialen Sprache in Deutschland, Stuttgart: Klett 1975, Bd. 2, S. 593-717.

Krufft, Hanno Walter: Geschichte der Architekturtheorie, München: Beck 1985.

Kurmann, Peter: »Französische Bischöfe als Auftraggeber und Stifter von Glasmalereien. Das Kunstwerk als Geschichtsquelle«, in: Zeitschrift für Kunstgeschichte 60 (1997), S. 429-450.

Le Corbusier: Vers une architecture, Paris: Ed. Crès 1924. 
Lillich, Meredith Parsons: »King Solomon in Bed, Archbishop Hincmar, the >Ordo of 1250 , and the Stained-Glass Program of the Nave of Reims Cathedral«, in: Speculum 80 (2005), S. 764-801.

Maurras, Charles: Anthinea. D'Athènes à Florence, Paris: Juven 1901, wieder abgedruckt in: Ders., Oeuvres capitales. Bd. 1: Sous le signe de Minerve, Paris: Flammarion 1954, S. 167-257.

Muhlack, Ulrich: Geschichtswissenschaft im Humanismus und in der Aufklärung. Die Vorgeschichte des Historismus, München: Beck 1991.

Nietzsche, Friedrich: »Unzeitgemäße Betrachtungen, zweites Stück: Vom Nutzen und Nachtheil der Historie für das Leben « [1874], in: Ders., Werke. Kritische Gesamtausgabe. 3. Abteilung, Bd. 1: Die Geburt der Tragödie. Unzeitgemäße Betrachtungen I-III (1872-1874), hg. v. Giorgio Colli u. Mazzino Montinari, Berlin/New York: De Gruyter 1972, S. $239-330$.

Oexle, Otto Gerhard/Rüsen, Jörn (Hg.): Historismus in den Kulturwissenschaften, Köln/Weimar/Wien: Böhlau 1996.

Oexle, Otto Gerhard: Geschichtswissenschaft im Zeichen des Historismus, Göttingen: Vandenhoeck \& Ruprecht 1996.

Palladio, Andrea: I quattro libri dell'architettura, hg. v. Licisco Magagnato u. Paola Marini, Mailand: Il Polifilo 1980 [1570].

Perrault, Charles: Parallèle des anciens et des modernes en ce qui regarde les arts et les sciences, eingeleitet von Hans Robert Jauss, München: Eidos 1964 .

Romantik und Restauration. Architektur in Bayern zur Zeit Ludwigs I. 18251848. Ausstellung der Architektursammlung der Technischen Universität München und des Münchner Stadtmuseums in Verbindung mit dem Zentralinstitut für Kunstgeschichte [München, Münchner Stadtmuseum, 27. Februar - 24. Mai 1987], hg. v. Winfried Nerdinger, München: Prestel 1987.

Rüsen, Jörn: Historik. Theorie der Geschichtswissenschaft, Köln: Böhlau 2013.

Schmale, Franz-Josef: Funktion und Formen mittelalterlicher Geschichtsschreibung. Eine Einführung, Darmstadt: Wissenschaftliche Buchgesellschaft 1993 .

Serlio, Sebastiano: Regole generali di architettura sopra le cinque maniere degli edifici, cio e thoscano, dorico, ionico, corinthio et composito, con gli essempi dell'antiquita, che, per la magior parte concordano con la dottrina di Vitruvio, Venedig: Marcolini da Forli 1537. 
Sot, Michel: Un historien et son église au Xe siècle: Flodoard de Reims, Paris: Fayard 1993.

Weber, Max: »Die >Objektivität< sozialwissenschaftlicher und sozialpolitischer Erkenntnis« [1904], in: Ders., Gesammelte Aufsätze zur Wissenschaftslehre, hg. v. Johannes Winckelmann, Tübingen: Mohr-Siebeck 1973 , S. 14 6-214.

Winckelmann, Johann Joachim: Geschichte der Kunst des Altertums, Dresden: Walther'sche Hofbuchhandlung 1764 .

Wittkau-Horgby, Annette: Historismus. Zur Geschichte des Begriffs und des Problems, Göttingen: Vandenhoeck \& Ruprecht 1994. 